\title{
ON CONSTRUCTING A MULTIDIMENSIONAL DIFFUSION PROCESS WITH A MEMBRANE LOCATED ON A GIVEN HYPERPLANE AND ACTING IN AN OBLIQUE DIRECTION
}

\author{
Mykola Portenko ${ }^{1}$, Bohdan Kopytko ${ }^{2}$ \\ ${ }^{I}$ Institute of Mathematics of Ukrainian National Academy of Science, Kyiv, Ukraine \\ ${ }^{2}$ Institute of Mathematics, Czestochowa University of Technology, Czestochowa, Poland \\ portenko@imath.kiev.ua, bohdan.kopytko@im.pcz.pl
}

\begin{abstract}
Using the methods of the theory of classical potentials, we have constructed a Feller semigroup of linear operators that generates a multidimensional diffusion process whose diffusion matrix is given by a sufficiently regular function and whose drift vector is given by a generalized function of the type of a $\delta$-function concentrated on a given hyperplane. Such process can serve as a mathematical model for describing the motion of a diffusing particle in a medium where a membrane is located on the hyperplane. The particle is receiving "a pulse of infinite intensity" at those instants of time when it is hitting the hyperplane. The direction of those pulses are determined by a vector field given on that hyperplane. It is important to emphasize that the trajectories of the process constructed are continuous.
\end{abstract}

\section{Introduction}

It is well known that the notion of a diffusion process in modern mathematics is intended to serve as a mathematical model for describing the motion of a diffusing particle, i.e. a small particle suspended in a liquid or a gas (Brownian motion, a phenomenon discovered by the English botanist Robert Brown in 1828). In short, that notion singles out a class of continuous Markov processes for which some local characteristics of the motion exist, namely, a drift vector and a diffusion matrix (operator) that respectively characterize the macroscopic and microscopic components of the motion.

An important problem is to develop some methods for constructing a diffusion process with its local characteristics previously indicated, i.e. vector-valued and matrix-valued functions are given and a diffusion process is to be constructed such that its drift vector and its diffusion matrix are represented respectively by the first and the second one of the two given functions. If those functions are sufficiently regular, one can make use of either the analytical method (the so-called Kolmogorov equation) or the method of stochastic differential equations in order to construct a diffusion process desired. Various results of the type are well known. 
It turns out, however, that a diffusion process can be constructed from some given functions (mentioned above) being extremely irregular. In particular, a function representing the drift vector can be a generalized one of the type of a $\delta$ function concentrated on a given surface (a function represented the diffusion matrix should be at the same time a "nice" one). Such a process describes the motion of a diffusing particle in a medium where a membrane is situated on the surface. The particle receives a pulse "of infinite intensity" every time it hits the surface. In [1] and [2] some results of the type are presented in the situation when the direction of the pulse coincides with the conormal vector to the surface. Using the ideas of [1] - [3], we constructed in our paper [4] the corresponding diffusion process in the situation when the direction of the pulse is determined by an arbitrary vector field on a given hyperplane while the diffusion matrix was there assumed to be an identity one. The aim of this paper is to generalize that result on the situation when the diffusion matrix is an arbitrary bounded, Hölder continuous and uniformly nondegenerated matrix-valued function.

The Feller semigroup of operators that determines the process desired is here constructed as a solution of some parabolic conjugation problem with oblique derivatives in one of the two conjugation conditions posed at the points of a fixed hyperplane. And the classical solvability of the problem is established here by the method of boundary integral equations with the use of a single-layer potential generated by an ordinary fundamental solution of a parabolic equation with Hölder continuous coefficients [3], [5], [6], [7, Ch. IV, §15], [8, Ch. V].

Notice that diffusion processes of the kind were constructed in [9], but the Pagni special single-layer potential [10] was used there instead of an ordinary one used in this paper. It should be mentioned that our problem was also investigated in [11] and [14] by the methods of stochastic analysis.

\section{Notations, the problem formulation, assumptions}

We will use the following notations: $\mathbb{R}^{d}$ - is a $d$-dimensional Euclidean space, $d \geq 2 ; x=\left(x_{1}, \ldots, x_{d}\right)$ is a point in $\mathbb{R}^{d}, x^{\prime}=\left(x_{1}, \ldots, x_{d-1}\right)$ is a point in $\mathbb{R}^{d-1}$ (by $x^{\prime}$ sometimes denoted a point of the form $\left.\left(x_{1}, \ldots, x_{d-1}, 0\right)\right) ;(x, y)=$ $=\sum_{i=1}^{d} x_{i} y_{i}, \quad$ if $\quad\{x, y\} \subset \mathbb{R}^{d} ; \quad\left(x^{\prime}, y^{\prime}\right)=\sum_{i=1}^{d-1} x_{i} y_{i}, \quad$ if $\quad\left\{x^{\prime}, y^{\prime}\right\} \subset \mathbb{R}^{d-1}$; $\mathcal{D}_{1}=\mathbb{R}_{-}^{d}=\left\{x \in \mathbb{R}^{d}: x_{d}<0\right\}, \mathcal{D}_{2}=\mathbb{R}_{+}^{d}=\left\{x \in \mathbb{R}^{d}: x_{d}>0\right\}, \mathcal{S}=\mathbb{R}^{d-1}$ is the boundary of the domains $\mathcal{D}_{l}, l \in\{1,2\}$, so that $\mathbb{R}^{d}=\mathcal{D}_{1} \cup \mathcal{S} \cup \mathcal{D}_{2} ; v\left(x^{\prime}\right)=$ $=\left(v_{i}\left(x^{\prime}\right)\right)_{i=1}^{d}, v_{i}\left(x^{\prime}\right)=0, i \in\{1, \ldots, d-1\}, v_{d}=1$ is a unit normal vector to $S$ at the point $x^{\prime}$, directed into the domain $\mathcal{D}_{2} ;(t, x)=\left(t, x^{\prime}, x_{d}\right)$ is a point of $\mathbb{R}^{d+1} ; T$ is a fixed positive number; $\mathbb{R}_{\infty}^{d+1}=(0, \infty) \times \mathbb{R}^{d} ; \mathbb{R}_{\infty}^{d}=(0, \infty) \times \mathbb{R}^{d-1}$; $\mathbb{R}_{T}^{d+1}=(0, T) \times \mathbb{R}^{d} ; \Omega^{(l)}=(0, \infty) \times \mathcal{D}_{l}, \Omega_{T}^{(l)}=(0, T) \times \mathcal{D}_{l}, l \in\{1,2\}$ is a domain in $\mathbb{R}^{d+1} ; \bar{Q}$ is the closure of the set $Q ; D_{t}^{1}=D_{t}=\frac{\partial}{\partial t}, D_{i}=\frac{\partial}{\partial x_{i}}, D_{i j}=\frac{\partial^{2}}{\partial x_{i} \partial x_{j}}$, $\{i, j\} \subset\{1, \ldots, d\}$ are the symbols of differentiation; $D_{t}^{r}$ and $D_{x}^{p}$ are the symbols of 
the partial derivative of the order $r$ with respect to $t$ and any partial derivative of the order $p$ with respect to $x$ respectively, where $r$ and $p$ are non-negative integers; $\nabla^{\prime}=\left(D_{1}, \ldots, D_{d-1}\right)$ is a spatial gradient; $\Delta_{x}^{\tilde{x}} f(\cdot, x)=f(\cdot, x)-f(\cdot, \tilde{x}), \Delta_{t}^{\tilde{t}} f(t, \cdot)=$ $=f(t, \cdot)-f(\tilde{t}, \cdot) ; \mathcal{B}\left(\mathbb{R}^{d}\right)$ is the Banach space of all real-valued bounded and measurable functions on $\mathbb{R}^{d}$ with the norm $\|\varphi\|=\sup _{\mathrm{x} \in \mathbb{R}^{\mathrm{d}}}|\varphi(x)| ; \mathcal{C}\left(\mathbb{R}^{d}\right)$ is the space of all real-valued continuous functions on $\mathbb{R}^{d} ; \mathcal{H}^{\lambda}\left(\mathbb{R}^{d}\right), \mathcal{H}^{\lambda}\left(\mathbb{R}^{d-1}\right)$, $\lambda \in(0,1)$ are spaces of Hölder continuous functions (see [7, Ch. I, $\S 1]$ ); $\mathcal{C}(\Omega)$ $(\mathcal{C}(\bar{\Omega}))$ is the set of all continuous functions on $\Omega(\bar{\Omega})$, where $\Omega$ is a subset of the region $\mathbb{R}_{\infty}^{d+1} ; \mathcal{C}^{1,2}(\Omega)\left(\mathcal{C}^{1,2}(\bar{\Omega})\right)$ is the set of all continuous functions on $\Omega(\bar{\Omega})$ that have continuous on $\Omega(\bar{\Omega})$ derivatives $D_{t}^{r}$ and $D_{x}^{p}, r=1, p \leq 2$. Everywhere below $C$ and $c$ there are some positive constants that do not depend on $(t, x)$ and as a rule their values are not important to us. Other notations will be explained as soon as they have arisen.

Suppose that a differentiable operator $L$ is given on $\mathbb{R}^{d}, d \geq 2$, such that it generates a homogeneous diffusion process

$$
L f(x)=\frac{1}{2} \sum_{i, j=1}^{d} b_{i j}(x) D_{i j} f(x)+\sum_{i=1}^{d} a_{i}(x) D_{i} f(x),
$$

where $b_{i j}(x)$ and $a_{i}(x)$ are real-valued bounded continuous functions on $\mathbb{R}^{d}$ and the matrix $b(x)=\left(b_{i j}(x)\right)_{i, j=1}^{d}$ is a symmetric nonnegative definite one. In the theory of diffusion processes the matrix $b(x)$ and the vector $a(x)=\left(a_{i}(x)\right)_{i=1}^{d}$ are called the diffusion matrix and the drift vector respectively (see [1]). Suppose now that some continuous bounded functions $\alpha_{i}\left(x^{\prime}\right), q_{j}\left(x^{\prime}\right)$ for $i \in\{1, \ldots, d-1\}$, $j \in\{1,2\}$ and $x^{\prime} \in S$ are given such that $q_{j}\left(x^{\prime}\right) \geq 0$ and $q_{1}(x)+q_{2}(x)>0$.

The problem is to construct a semigroup of linear operators $T_{t}, t \geq 0$, such that $T_{t}: \mathcal{C}\left(\mathbb{R}^{d}\right) \cap \mathcal{B}\left(\mathbb{R}^{d}\right) \rightarrow \mathcal{C}\left(\mathbb{R}^{d}\right) \cap \mathcal{B}\left(\mathbb{R}^{d}\right)$ and the function $u(t, x, \varphi)$ defined for $t \geq 0, x \in \mathbb{R}^{d}$ and $\varphi \in \mathcal{C}\left(\mathbb{R}^{d}\right) \cap \mathcal{B}\left(\mathbb{R}^{d}\right)$ by the equality $u(t, x, \varphi)=T_{t} \varphi(x)$ (we will write down $u(t, x)$ instead of $u(t, x, \varphi))$ is a solution to the following parabolic conjugation problem:

$$
\begin{gathered}
D_{t} u(t, x)=L u(t, x), \quad(t, x) \in \Omega^{(l)}, l \in\{1,2\}, \\
u(0, x)=\varphi(x), \quad x \in \mathbb{R}^{d}, \\
u\left(t, x^{\prime}, 0-\right)=u\left(t, x^{\prime}, 0+\right), \quad\left(t, x^{\prime}\right) \in \mathbb{R}_{\infty}^{d}, \\
L_{0} u\left(t, x^{\prime}, 0\right) \equiv \sum_{i=1}^{d-1} \alpha_{i}\left(x^{\prime}\right) D_{i} u\left(t, x^{\prime}, 0\right)-q_{1}\left(x^{\prime}\right) D_{d} u\left(t, x^{\prime}, 0-\right) \\
+q_{2}\left(x^{\prime}\right) D_{d} u\left(t, x^{\prime}, 0+\right)=0, \quad\left(t, x^{\prime}\right) \in \mathbb{R}_{\infty}^{d} .
\end{gathered}
$$


The equalities (2)-(5) mean that the semigroup $T_{t}, t \geq 0$, corresponds to a Feller process in $\mathbb{R}^{d}$ such that its part in the domain $D_{1} \cup D_{2}$ is a diffusion process generated by the operator $L$ and its behaviour at the points of the boundary $S$ (where a membrane is situated) is determined by a particular case of the general Ventsell boundary condition [12]. Namely, our particle, after reaching a point on $S$, can be either reflected into the domain where it has come from or can penetrate into the opposite domain; besides it can be drifted along the hyperplane. Combining all these possibilities, we can say that the membrane (which is situated on $S$ ) affects our particle in an oblique direction (see Theorem 2 below).

We are going to construct a bounded (in the spatial variable) classical solution of the problem (2)-(5), such that the function $u(t, x)$ belongs to $\mathcal{C}^{1,2}\left(\Omega^{(l)}\right) \cap$ $\mathcal{C}\left(\overline{\mathbb{R}_{\infty}^{d+1}}\right)$.

\section{Solving the parabolic conjugation problem}

In order to solve the problem (2)-(5) we apply the method of boundary integral equations. Suppose that the coefficients of the operator $L$ and $L_{0}$ satisfy following conditions:

a) $\left(\exists \delta_{0}>0\right)\left(\forall x \in \mathbb{R}^{d}, \forall \Theta \in \mathbb{R}^{d}\right),(b(x) \Theta, \Theta) \geq \delta_{0}|\Theta|^{2}$;

b) $b_{i j}, a_{i} \in \mathcal{H}^{\lambda}\left(\mathbb{R}^{d}\right), \lambda \in(0,1),\{\mathrm{i}, \mathrm{j}\} \subset\{1, \ldots, \mathrm{d}\}$;

c) $q_{l}\left(x^{\prime}\right) \geq 0, \quad x^{\prime} \in \mathbb{R}^{d-1}, \quad l \in\{1,2\} \quad$ and $\left(\exists q_{0}>0\right)\left(\forall x^{\prime} \in \mathbb{R}^{d-1}\right), \quad q_{1}\left(x^{\prime}\right)+$ $q_{2}\left(x^{\prime}\right) \geq q_{0}$

d) $\alpha_{i}, q_{l} \in \mathcal{H}^{\lambda}\left(\mathbb{R}^{d-1}\right), i \in\{1, \ldots, d-1\}, l \in\{1,2\}$.

Let $g(t, x, y)\left(t>0, x \in \mathbb{R}^{d}, y \in \mathbb{R}^{d}\right)$ denote the fundamental solution (f.s.) of the operator $D_{t}-L$ (see [1], [2], [5], [7], [8]):

$$
g(t, x, y)=g_{0}(t, x, y)+g_{1}(t, x, y)
$$

where

$$
\begin{aligned}
& g_{0}(t, x, y)=g_{0}^{(y)}(t, x-y) \\
& \quad=(2 \pi t)^{-\frac{d}{2}}(\operatorname{det} b(y))^{-\frac{1}{2}} \exp \left(-\frac{1}{2 t}\left(b^{-1}(y)(y-x), y-x\right)\right),
\end{aligned}
$$

$g_{1}$ is a term whose singularity, as $t \rightarrow 0+$, is weaker than that of $g_{0}$. The following inequalities are true for the functions $g$ and $g_{1}$ for $2 r+p \leq 2, t \in(0, T]$ and $\{x, y\} \subset \mathbb{R}^{d}:$

$$
\begin{gathered}
\left|D_{t}^{r} D_{x}^{p} g(t, x, y)\right| \leq C t^{-\frac{d+2 r+p}{2}} \exp \left(-c \frac{|y-x|^{2}}{t}\right), \\
\left|D_{t}^{r} D_{x}^{p} g_{1}(t, x, y)\right| \leq C t^{-\frac{d+2 r+p-\lambda}{2}} \exp \left(-c \frac{|y-x|^{2}}{t}\right) .
\end{gathered}
$$


As a function of $(t, x)$, the principal part $g_{0}$ of f.s. has the derivatives in $t$ and $x$ of any order and the inequalities (7) and (8) are satisfied by them for $t>0$ and for all non-negative integers $r$ and $p$. Besides, the function $g_{0}^{(y)}(t, z)$ and its derivatives in $t$ and $z$ are Hölder continuous in the argument $y$ (see inequality (11.4) in [7, Ch. IV]). We will also use the estimates (13.2) and (13.3) from [7, Ch. IV], which will be applied to the differences $\Delta_{x}^{\tilde{x}}\left(D_{t}^{r} D_{x}^{p} g(t, x, y)\right)$ and $\Delta_{t}^{\tilde{t}}\left(D_{t}^{r} D_{x}^{p} g(t, x, y)\right)$ respectively.

Let us consider the following integrals: the Poisson heat potential

$$
u_{0}(t, x)=\int_{\mathbb{R}^{d}} g(t, x, y) \varphi(y) d y, \quad(t, x) \in \mathbb{R}_{\infty}^{d+1}
$$

and the parabolic single-layer potential

$$
u_{1}(t, x)=\int_{0}^{t} d \tau \int_{\substack{\mathbb{R}^{d-1} \\(t, x) \in \mathbb{R}_{\infty}^{d+1}}} g\left(t-\tau, x, y^{\prime}\right) V\left(\tau, y^{\prime}\right) d y^{\prime},
$$

where $\varphi$ and $V$ are some given functions. The estimates imply that under some assumptions on the densities $\varphi$ and $V$ the functions $u_{0}$ and $u_{1}$ are continuous on $\overline{\mathbb{R}_{\infty}^{d+1}}$, satisfy the equation (2) and also the initial conditions $u_{0}(0, x)=\varphi(x)$ and $u_{1}(0, x)=0$. The behaviour of $D_{x} u_{1}(t, x)$, as $x \rightarrow x_{0} \in S$, is characterized in [1], [2], [6] - [8]. The most important property of those derivatives is known as the theorem on the jump of the conormal derivative of a single-layer potential. Denote by $\frac{\partial u_{1}\left(t, x_{0}-\right)}{\partial N\left(x_{0}\right)}$ and $\frac{\partial u_{1}\left(t, x_{0}+\right)}{\partial N\left(x_{0}\right)}$ the boundary values of the conormal derivative $\frac{\partial u_{1}(t, x)}{\partial N\left(x_{0}\right)}$ as $x$ tends to $x_{0} \in S$ from the domains $D_{1}$ and $D_{2}$ respectively. The theorem mentioned above says: if the function $V\left(t, x^{\prime}\right)$ is continuous on $\mathbb{R}_{\infty}^{d}$, bounded in spatial variables and has an integrable singularity in the variable $t$, then for any point $x_{0} \in S$ and $t>0$ the following equality

$$
\begin{aligned}
\frac{\partial u_{1}\left(t, x_{0} \mp\right)}{\partial N\left(x_{0}\right)}= & \int_{0}^{t} d \tau \int_{\mathbb{R}^{d-1}} \frac{\partial g\left(t-\tau, x_{0}, y^{\prime}\right)}{\partial N\left(x_{0}\right)} V\left(\tau, y^{\prime}\right) d y^{\prime} \\
& \pm V\left(t, x_{0}\right)
\end{aligned}
$$

holds true.

The integral on the right hand side of (11) is called the direct value of the conormal derivative of the single-layer potential (10). Its existence follows from the inequality

$$
\left|\frac{\partial g\left(t-\tau, x_{0}, y^{\prime}\right)}{\partial N\left(x_{0}\right)}\right| \leq C(t-\tau)^{-\frac{d+1-\lambda}{2}} \exp \left(-c \frac{\left|x_{0}-y^{\prime}\right|^{2}}{t-\tau}\right)
$$

that is held for $t \in(0, T],\left\{x_{0}, y^{\prime}\right\} \subset S$ with some constants $C$ and $c$. 
Another property concerns the behaviour of the derivatives of the function $u_{1}$ in the directions tangent to $S$ at the point $x_{0}$ (in the case under consideration they are the partial derivatives $\left.D_{i} u_{1}(t, x), i \in\{1, \ldots, d-1\}\right)$. It is known that in the case of the function $V$ being Hölder continuous in the spatial variables, the tangent derivatives of $u_{1}$ as well as the function $u_{1}$ itself are continuous in $x$ in any neighbourhood of $S$.

The problem (2)-(5) will be reduced to solving a Volterra integral equation. We are going to look for the solution $u(t, x)$ of the problem (2)-(5) in the following form:

$$
u(t, x)=u_{0}(t, x)+u_{1}(t, x),(t, x) \in \mathbb{R}_{\infty}^{d+1},
$$

where $u_{0}$ and $u_{1}$ are defined by the formulas (9) and (10) respectively; the function $\varphi$ in (9) coincides with the initial function in (3); the density $V$ in (10) is an unknown function. In order to determine this, we use the conjugation condition (5) that can be written in the following form:

$$
\begin{aligned}
L_{0} u\left(t, x^{\prime}, 0\right) \equiv & \sum_{i=1}^{d-1}\left(\alpha_{i}\left(x^{\prime}\right)-\frac{q_{2}\left(x^{\prime}\right)-q_{1}\left(x^{\prime}\right)}{b_{d d}\left(x^{\prime}\right)} b_{i d}\left(x^{\prime}\right)\right) \\
& \times D_{i} u\left(t, x^{\prime}, 0\right)-\frac{q_{1}\left(x^{\prime}\right)}{b_{d d}\left(x^{\prime}\right)} \frac{\partial u\left(t, x^{\prime}, 0-\right)}{\partial N\left(x^{\prime}\right)} \\
& +\frac{q_{2}\left(x^{\prime}\right)}{b_{d d}\left(x^{\prime}\right)} \frac{\partial u\left(t, x^{\prime}, 0+\right)}{\partial N\left(x^{\prime}\right)}=0,\left(t, x^{\prime}\right) \in \mathbb{R}_{\infty}^{d} .
\end{aligned}
$$

This relation together with (11) imply the following relation for the $V\left(t, x^{\prime}\right)$ :

$$
\begin{gathered}
V\left(t, x^{\prime}\right)=\int_{0}^{t} d \tau \int_{\mathbb{R}^{d-1}} K\left(t-\tau, x^{\prime}, y^{\prime}\right) V\left(\tau, y^{\prime}\right) d y^{\prime} \\
+\Psi\left(t, x^{\prime}\right), \quad\left(t, x^{\prime}\right) \in \mathbb{R}_{\infty}^{d}
\end{gathered}
$$

where:

$$
\begin{gathered}
K\left(t-\tau, x^{\prime}, y^{\prime}\right)=\left(\alpha_{0}^{\prime}\left(x^{\prime}\right), \nabla_{x^{\prime}}^{\prime} g\left(t-\tau, x^{\prime}, y^{\prime}\right)\right)+q\left(x^{\prime}\right) \frac{\partial g\left(t-\tau, x^{\prime}, y^{\prime}\right)}{\partial N\left(x^{\prime}\right)}, \\
\alpha_{0}^{\prime}\left(x^{\prime}\right)=\left(\alpha_{i}^{(0)}\left(x^{\prime}\right)\right)_{i=1}^{d-1}, \quad \alpha_{i}^{(0)}\left(x^{\prime}\right)=\frac{b_{d d}\left(x^{\prime}\right) \alpha_{i}\left(x^{\prime}\right)}{q_{1}\left(x^{\prime}\right)+q_{2}\left(x^{\prime}\right)}-q\left(x^{\prime}\right) b_{i d}\left(x^{\prime}\right) \\
q\left(x^{\prime}\right)=\frac{q_{2}\left(x^{\prime}\right)-q_{1}\left(x^{\prime}\right)}{q_{1}\left(x^{\prime}\right)+q_{2}\left(x^{\prime}\right)}, \quad \Psi\left(t, x^{\prime}\right)=\frac{b_{d d}\left(x^{\prime}\right) L_{0} u_{0}\left(t, x^{\prime}, 0\right)}{q_{1}\left(x^{\prime}\right)+q_{2}\left(x^{\prime}\right)} .
\end{gathered}
$$


The equality (15) is a Volterra integral equation of the second kind. It follows from (7), (8), (12) and also from the estimates (13.2), (13.3) in [7, Ch. IV] that the kernel $K$ and the function $\Psi$ satisfy for $0 \leq \tau<t \leq T,\left\{x^{\prime}, y^{\prime}\right\} \in \mathbb{R}^{d-1}$ and $t \in(0, T],\left\{x^{\prime}, \tilde{x}^{\prime}\right\} \subset \mathbb{R}^{d-1}$ the following inequalities respectively:

$$
\begin{gathered}
\left|K\left(t-\tau, x^{\prime}, y^{\prime}\right)\right| \leq C(t-\tau)^{-\frac{d+1}{2}} \exp \left(-c \frac{\left|x^{\prime}-y^{\prime}\right|^{2}}{t-\tau}\right), \\
\left|\Psi\left(t, x^{\prime}\right)\right| \leq C\|\varphi\| t^{-\frac{1}{2}}, \\
\left|\Delta_{x^{\prime}}^{\tilde{x}^{\prime}} \Psi\left(t, x^{\prime}\right)\right| \leq C\|\varphi\| t^{-\frac{1+\lambda}{2}}\left|x^{\prime}-\tilde{x}^{\prime}\right|^{\lambda} .
\end{gathered}
$$

As inequality (16) shows, the singularity of the kernel $K$ is such that it does not allow us to apply the method of successive approximations to equation (15). It means that this equation should be regularized. With that end in view we introduce an integro-differential operator $\mathcal{E}$ that acts on a function $\Psi$ according to the following rule:

$$
\begin{aligned}
\mathcal{E}\left(t, x^{\prime}\right) \Psi=\sqrt{\frac{2}{\pi}} & \left(\frac{\partial}{\partial t} \int_{0}^{t} d \tau \int_{\mathbb{R}^{d-1}} \Psi\left(\tau, y^{\prime}\right) d y^{\prime}\right. \\
& \times \int_{0}^{\infty}(t-\tau)^{-\frac{1}{2}} \exp \left(-\frac{u^{2}}{2(t-\tau)}\right) \\
& \left.\times h\left(\hat{t}-\tau, x^{\prime}+\tilde{\alpha}^{\prime}\left(x^{\prime}\right) u, y^{\prime}\right) d u\right)\left.\right|_{\hat{t}=t},
\end{aligned}
$$

where $h\left(t, x^{\prime}, y^{\prime}\right)\left(t>0, x^{\prime} \in \mathbb{R}^{d-1}, y^{\prime} \in \mathbb{R}^{d-1}\right)$ stands for f.s. of the following uniformly parabolic operator with Hölder continuous coefficients

$$
\begin{gathered}
D_{t}-\frac{1}{2} \sum_{i, j=1}^{d-1} h_{i j}\left(x^{\prime}\right) D_{i j}, \\
h_{i j}\left(x^{\prime}\right)=b_{i j}\left(x^{\prime}\right)-\frac{b_{i d}\left(x^{\prime}\right) b_{j d}\left(x^{\prime}\right)}{b_{d d}\left(x^{\prime}\right)},
\end{gathered}
$$

and $\tilde{\alpha}^{\prime}\left(x^{\prime}\right)=\left(\tilde{\alpha}_{i}\left(x^{\prime}\right)\right)_{i=1}^{d-1}$ denotes the vector whose coordinates are expressed by the coordinates of the vector $\alpha_{0}^{\prime}\left(x^{\prime}\right)$ as follows $\tilde{\alpha}_{i}\left(x^{\prime}\right)=\left(b_{d d}\left(x^{\prime}\right)\right)^{-\frac{1}{2}} \alpha_{i}^{(0)}\left(x^{\prime}\right)$.

If the function $\Psi$ in (19) possesses the same properties as the function $\Psi$ in (15), then putting $\widetilde{\Psi}\left(t, x^{\prime}\right)=\mathcal{E}\left(t, x^{\prime}\right) \Psi$, we arrive at the relation 


$$
\begin{aligned}
\widetilde{\Psi}\left(t, x^{\prime}\right)=\Psi(t, & \left.x^{\prime}\right)+\frac{1}{\sqrt{2 \pi}} \int_{0}^{t}(t-\tau)^{-\frac{3}{2}} d \tau \\
\times & \int_{\mathbb{R}^{d-1}}\left(\int_{0}^{\infty}\left(1-\frac{u^{2}}{t-\tau}\right) \exp \left(-\frac{u^{2}}{2(t-\tau)}\right)\right. \\
\times & \left.h\left(t-\tau, x^{\prime}+\tilde{\alpha}^{\prime}\left(x^{\prime}\right) u, y^{\prime}\right) d u\right)\left(\Psi\left(\tau, y^{\prime}\right)\right. \\
& \left.-\Psi\left(\tau, x^{\prime}\right)\right) d y^{\prime}, \quad\left(t, x^{\prime}\right) \in \mathbb{R}_{\infty}^{d} .
\end{aligned}
$$

From this equality, taking into account the property of f.s., the estimates (17), (18), and using the following inequality:

$$
\begin{aligned}
\exp \left(-c \frac{u^{2}}{t-\tau}\right) & \exp \left(-c \frac{\left|x^{\prime}+\tilde{\alpha}^{\prime}\left(x^{\prime}\right) u-y^{\prime}\right|^{2}}{t-\tau}\right) \\
& \leq \exp \left(-c^{\prime} \frac{u^{2}}{t-\tau}\right) \exp \left(-c^{\prime} \frac{\left|x^{\prime}-y^{\prime}\right|^{2}}{t-\tau}\right)
\end{aligned}
$$

valid for all $\left\{x^{\prime}, y^{\prime}\right\} \in \mathbb{R}^{d-1}, u \in \mathbb{R}^{1}, 0 \leq \tau<t$, with an arbitrary positive constant $c$ and some constant $c^{\prime}, 0<c^{\prime}<c$, we obtain that the function $\widetilde{\Psi}\left(t, x^{\prime}\right)$ as well as the function $\Psi\left(t, x^{\prime}\right)$ is continuous in the region $\left(t, x^{\prime}\right) \in \mathbb{R}_{d}^{\infty}$, and it satisfies the inequalities (17), (18).

Further, by the analogy to that of [4] we prove that the equation (15) can be transformed by the operator $\mathcal{E}$ (applied to both sides of (15)) into the following equivalent Volterra equation of the second kind:

$$
\begin{gathered}
V\left(t, x^{\prime}\right)=\int_{0}^{t} d t \int_{\mathbb{R}^{d-1}} \widetilde{K}\left(t-\tau, x^{\prime}, y^{\prime}\right) V\left(\tau, y^{\prime}\right) d y^{\prime}+\widetilde{\Psi}\left(t, x^{\prime}\right),\left(t, x^{\prime}\right) \in \mathbb{R}_{\infty}^{d}, \\
\widetilde{\Psi}\left(t, x^{\prime}\right)=\varepsilon\left(t, x^{\prime}\right) \Psi
\end{gathered}
$$

and for the kernel $\widetilde{K}\left(t-\tau, x^{\prime}, y^{\prime}\right)$ for $0 \leq \tau<t \leq T,\left\{x^{\prime}, y^{\prime}\right\} \subset \mathbb{R}^{d-1}$ the following inequality

$$
\left|\widetilde{K}\left(t-\tau, x^{\prime}, y^{\prime}\right)\right| \leq C(t-\tau)^{-\frac{d+1-\lambda}{2}} \exp \left(-c \frac{\left|x^{\prime}-y^{\prime}\right|^{2}}{t-\tau}\right)
$$

holds true.

This inequality allows us to apply the method of successive approximations to the equation (21) and, as a consequence, we obtain the function $V$. In addition, we establish that the solution $V$ has the same properties as the function $\widetilde{\Psi}$, i.e. $V$ is continuous in the region $\left(t, x^{\prime}\right) \in \mathbb{R}_{\infty}^{d}$ and it satisfies the inequalities (17), (18). We have from this that the function $u(t, x)$ defined by the formulas (13), (21) is a classical solution of the problem that in any region of the form $\overline{\mathbb{R}}_{T}^{d+1}$ satisfies the estimate 


$$
|u(t, x)| \leq C\|\varphi\| .
$$

As for the uniqueness of the solution constructed, it can be deduced from the results of [13]. We have thus proved the following assertion.

Theorem 1. Let the coefficients of the operators $L$ and $L_{0}$ satisfy the conditions a)-d), and let the initial function $\varphi$ in (3) be continuous and bounded. Then the parabolic conjugation problem (2)-(5) has the unique classical solution that is defined by the formulas (13), (21) and satisfies the inequality (23).

\section{Constructing the diffusion process}

The Theorem 1 implies that we can define a semigroup of linear operators $T_{t}$, $t \geq 0$, whose action on a function $\varphi \in \mathcal{B}\left(\mathbb{R}^{d}\right) \cap \mathcal{C}\left(\mathbb{R}^{d}\right)$ is given by the formula $T_{t} \varphi(x)=u(t, x)$, where the function $u(t, x)$ is given by the formulas (13), (21). Using the well-known properties of potentials, and also the maximum principle for parabolic equations, we can now verify that the constructed semigroup satisfies the following additional properties:

$\left.1^{\circ}\right)$ if $\varphi_{n} \in \mathcal{B}\left(\mathbb{R}^{d}\right) \cap \mathcal{C}\left(\mathbb{R}^{d}\right)$ for $n=1,2, \ldots, \sup _{n}\left\|\varphi_{n}\right\|<\infty$ and for all $x \in \mathbb{R}^{d}$ the relation $\lim _{n \rightarrow \infty} \varphi_{n}(x)=\varphi(x)$ is true, $\varphi \in \mathcal{B}\left(\mathbb{R}^{d}\right) \cap \mathcal{C}\left(\mathbb{R}^{d}\right)$, then for all $t \geq 0, x \in \mathbb{R}^{d}$, the relation $\lim _{n \rightarrow \infty} T_{t} \varphi_{n}(x)=T_{t} \varphi(x)$ is valid;

$\left.2^{\circ}\right) T_{t} \varphi(x) \geq 0$ for all $t \geq 0, x \in \mathbb{R}^{d}$, if only the function $\varphi$ is non-negative;

$\left.3^{\circ}\right)\left\|T_{t}\right\| \leq 1$ for all $t \geq 0$.

These properties of the semigroup $T_{t}$ imply the existence of a transition probability $P(t, x, d y)$ in $\mathbb{R}^{d}$ such that

$$
T_{t} \varphi(x)=\int_{\mathbb{R}^{d}} P(t, x, d y) \varphi(y) .
$$

It can be established that a Feller process in $\mathbb{R}^{d}$ with this transition probability can be constructed in such a way that its trajectories are continuous. Some immediate calculations show that the local characteristics for this process exist in the following sense:

$$
\begin{aligned}
& \lim _{t \downarrow 0} \frac{1}{t} \int_{\mathbb{R}^{d}} \varphi(x)\left(\int_{\mathbb{R}^{d}}(y-x, \Theta) P(t, x, d y)\right) d x \\
& \quad=\int_{\mathbb{R}^{d}} \varphi(y)(a(y), \Theta) d y+\int_{\mathbb{R}^{d-1}} \varphi\left(y^{\prime}, 0\right)\left(\hat{\alpha}\left(y^{\prime}\right), \Theta\right) d y^{\prime}, \\
& \lim _{t \downarrow 0} \frac{1}{t} \int_{\mathbb{R}^{d}} \varphi(x)\left(\int_{\mathbb{R}^{d}}(y-x, \Theta)^{2} P(t, x, d y)\right) d x=\int_{\mathbb{R}^{d}} \varphi(y)(b(y) \Theta, \Theta) d y,
\end{aligned}
$$

where $\Theta \in \mathbb{R}^{d}, \varphi$ is an arbitrary continuous compactly supported function on $\mathbb{R}^{d}$, 


$$
\begin{gathered}
\hat{\alpha}\left(y^{\prime}\right)=\left(\hat{\alpha}_{i}\left(y^{\prime}\right)\right)_{i=1^{\prime}}^{d} \\
\hat{\alpha}_{i}\left(y^{\prime}\right)=\frac{b_{d d}\left(y^{\prime}\right) \alpha_{i}\left(y^{\prime}\right)}{q_{1}\left(y^{\prime}\right)+q_{2}\left(y^{\prime}\right)}, \quad i \in\{1, \ldots, d-1\}, \\
\hat{\alpha}_{d}\left(y^{\prime}\right)=b_{d d}\left(y^{\prime}\right) q\left(y^{\prime}\right) .
\end{gathered}
$$

The equalities (24), (25) mean that the drift vector and the diffusion matrix for the process constructed are respectively as follows

$$
a(x)+\hat{\alpha}\left(x^{\prime}\right) \delta_{s}(x) \text { and } b(x),
$$

where $\delta_{S}(x)$ is a generalized function on $\mathbb{R}^{d}$ whose action on a test function consists in integrating the latter one over $S$.

As a result we have the following statement.

Theorem 2. If the conditions of the Theorem 1 are fulfilled then the semigroup of linear operators constructed by solving the conjugation problem (2)-(5) corresponds to a continuous Feller process in $\mathbb{R}^{d}$ whose local characteristics exist in sense that the equalities (24) and (25) hold true.

\section{References}

[1] Portenko N.I., Generalized Diffusion Processes, Kyiv, 1982; English transl., Amer. Math. Soc., Providence, RI, 1990.

[2] Portenko M.I., Diffusion Processes in Media with Membranes, Institute of Mathematics, of Ukrainian National Acad. of Sci., Kyiv 1995 (in Ukrainian).

[3] Baderko E.A., Solution of a problem with oblique derivative for parabolic equation by method of boundary integral equations, Differential Equations 1971, 25, 1, 14-20 (in Russian).

[4] Kopytko B.I., Portenko M.I., On a Multidimensional Brownian Motion with a Membrane Located on a Hyperplane and Acting in an Oblique Direction, Probability Theory and Mathematical Statistics, Proceedings, Institute of Mathematics of Ukrainian National Acad. of Sci., Kyiv 2002, 73-84.

[5] Pogorzelski W., Étude de la solution fondamental de l'equation parabolique, Richerche di Mat. 1956, 5, 25-27.

[6] Pogorzelski W., Study of integrals of parabolic equation and boundary problems in an unbounded domain, Math. Coll. 1959, 47, 4(89), 397-430 (in Russian).

[7] Ladyzhenskaya O.A., Solonnikov V.A., Ural'tseva N.N., Linear and Quasilinear Equations of Parabolic Type, Nauka, Moscow 1967 (in Russian).

[8] Friedman A., Partial Differential Equations of Parabolic Type, Prentice-Hall, Englewood Clifs, N.J. 1964.

[9] Kopytko B.I., Construction of the diffusion process with generalized drift vector by means of solution some conjugation problem for the second-order parabolic type equation, Random Oper. Stoch. Eqs., 1984, 2, 1, 33-38.

[10] Pagni M., Su un problema al contorno tipico per l'equazione del calore, Ann. Sc. Norm. Super. Di Pisa 1957, (3), 2, 1-2, 73-115. 
[11] Anulova S.V., Diffusion processes: discontinuous coefficients, degenerate diffusion, Randomized Drift, DAN USSR 1981, 260, 5, 1036-1040 (in Russian).

[12] Wentzel A.D., On boundary conditions for multidimensional diffusion processes, Theor. Prob. and Appl. 1959, 2, 5, 172-185 (in Russian).

[13] Kamynin L.I., Maslennikova V.N., On maximum principle for parabolic equation with discontinuous coefficients, Siberian Math. Journal 1961, 2, 3, 384-399 (in Russian).

[14] Zaitseva L.L., On a probabilistic approach to the construction of the generalized diffusion processes, Theory of Stochastic Processes 2000, 6(22), 1-2, 141-146. 
